University of Wollongong

Research Online

Faculty of Informatics - Papers (Archive)

Faculty of Engineering and Information

Sciences

18-3-2005

\title{
Virtual cochlear implant insertion for medical education
}

C. Todd

University of Wollongong, cath@uow.edu.au

F. Naghdy

University of Wollongong, fazel@uow.edu.au

Follow this and additional works at: https://ro.uow.edu.au/infopapers

Part of the Physical Sciences and Mathematics Commons

\section{Recommended Citation}

Todd, C. and Naghdy, F.: Virtual cochlear implant insertion for medical education 2005.

https://ro.uow.edu.au/infopapers/117

Research Online is the open access institutional repository for the University of Wollongong. For further information contact the UOW Library: research-pubs@uow.edu.au 


\title{
Virtual cochlear implant insertion for medical education
}

\author{
Abstract \\ A surgical simulator has been developed for the purpose of training otologists in cochlear implantation. \\ The simulation provides real-time visual and haptic feedback during implant insertion into the human \\ Scala Tympani (ST). The benefits and possible outcomes for this type of simulator are presented. \\ Methods for model generation are discussed, for anatomical and prosthetic structures used in the \\ simulation. Development of the interactive model with force-feedback is presented, with results.

\section{Keywords} \\ biomedical education, ear, force feedback, haptic interfaces, interactive devices, medical computing, \\ prosthetics, training, virtual reality \\ Disciplines \\ Physical Sciences and Mathematics

\section{Publication Details} \\ This paper originally appeared as: Todd, C and Naghdy, F, Virtual cochlear implant insertion for medical \\ education, First Joint Eurohaptics Conference and Symposium on Haptic Interfaces for Virtual \\ Environment and Teleoperator Systems, 18-20 March 2005, 505-506. Copyright IEEE 2005.
}




\title{
Virtual Cochlear Implant Insertion for Medical Education
}

\author{
Catherine Todd Fazel Naghdy \\ School of Electrical, Computer and Telecommunications Engineering \\ Faculty of Informatics, University of Wollongong, Australia \\ E-mail: cath@elec.uow.edu.au,fazel@uow.edu.au
}

\begin{abstract}
A surgical simulator has been developed for the purpose of training otologists in cochlear implantation. The simulation provides real-time visual and haptic feedback during implant insertion into the human Scala Tympani (ST). The benefits and possible outcomes for this type of simulator are presented. Methods for model generation are discussed, for anatomical and prosthetic structures used in the simulation. Development of the interactive model with force-feedback is presented, with results.
\end{abstract}

\section{Introduction}

A clinically valid surgical simulator is being developed to supplement current methods for training otologists to perform cochlear implant (CI) insertion. The proposed system will provide a safe, cost-effective training environment in which the patient is not at risk. Patient-specific models will allow for individual variances in cochlear morphology, proving beneficial for pre- and post- operative planning. The system will offer quantitative evaluation of forces during CI insertion into the ST, real-time electrode array location, insertion depth measurements and trauma analysis. Information of this type will assist in surgeon technique, force administration during implantation and assist in electrode array design improvements.

\section{Model of the Cochlear}

In this work, a first approximation of the cochlear is derived from CT. ANALYZE (AnalyzeDirect) [1] and the Visualization Toolkit (VTK) with Python are used for semi-automatic extraction of the cochlear. The VRML surface description is rendered with Reachin API (Reachin Technologies AB). The model contains a single inner chamber of the cochlear spiral.
A second, final model is derived from measured data [2-5] to form a parametric, reproducible model of the ST. In this model, the ST is separate from the Scala Vestibuli (SV) cavity (as desired for CI insertion). Measurements used to form the model include height and width of ST cross-sections, cochlear length, radial displacement about the modiolar axis, ST height variation from base to apex and intra-cochlear tilt. Parameters required for patient-specific model recreation include height and width data of the first ST cross-section, and cochlear length. Three-dimensional surfaces for the ST (Fig. 1), BM, Contour array and stylet are created using ANSYS (Leap Australia).

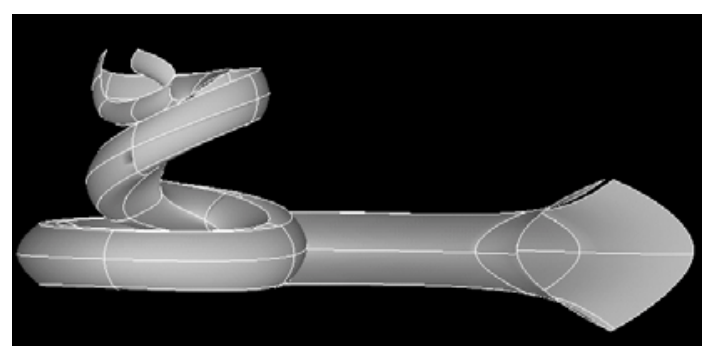

Fig. 1. Model of the ST generated in ANSYS

\section{Simulator Development}

\subsection{Method}

Reachin API is used for simulator development. It enables both visual and haptic rendering of a scene, with update rates of $30 \mathrm{~Hz}$ for the scene-graph (graphics) loop and $1000 \mathrm{~Hz}$ for the real-time (haptic) loop. VRML surface descriptions produced using ANSYS were scaled and visually rendered in the Reachin API, in the one scene-graph. Surface properties were added to the anatomical structures using SimpleSurface nodes, to provide haptic feedback between device tip and model. System features were programmed using VRML, Python and $\mathrm{C}++$. 


\subsection{System Features}

Following preliminary object visualisation, the second phase in simulator development was to make the simulation more dynamic. Python script was written to enable user-induced changes in object orientation and translation, as well as contour and stylet mobility about the scene. By pressing/releasing the haptic device button, the array and accompanying stylet could be moved precisely about the screen, with correct orientation and final placement.

A variety of tools was created, including Jeweller's Forceps, to interact with the virtual objects. Tool selection is via the keyboard as well as the haptic device button, but keyboard functionality may be replaced with button selection at the Graphical User Interface (GUI), using the haptics device tip.

Stylet withdrawal has been realised using both a right-angled hook through interaction with the stylet loop, and via the keyboard. Both methods allow for smooth stylet removal. With the hook selected, the ForceTorqueGroup node is used to facilitate stylet withdrawal. Scene magnification allows the practising surgeon to zoom in on the small cochlear structure, at selectable levels of magnification.

Collision detection and response between the array tip and ST has been implemented; full-body collision is work in progress. The Reachin API custom CollidingController node has been adapted to provide this functionality, yet there are instabilities (maximum force exertion, $8.5 \mathrm{~N}$, is exceeded) during collisions when object scale is significantly small $(<0.02 \mathrm{~m})$. Haptic device tip position and force outputs during collisions are shown on the GUI (refer Fig. 2). Surface properties of the ST and BM require further investigation, in order to provide the user with realistic force scaling and reflection. Currently the SimpleSurface node is utilised.

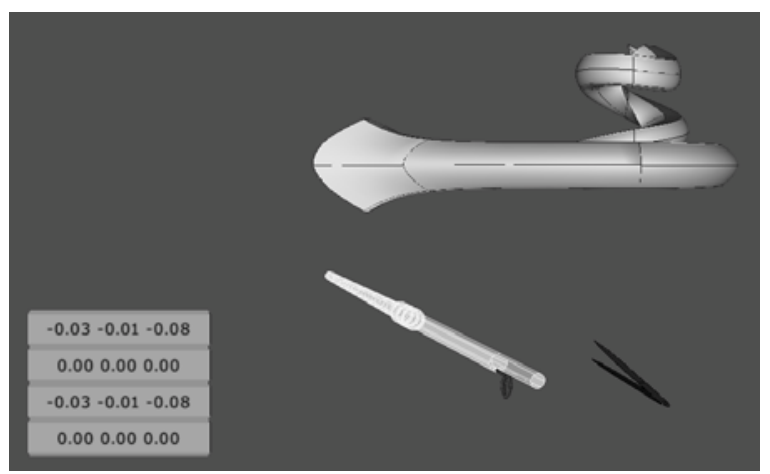

Fig. 2. Current GUI for CI simulator; model includes ST, BM, Contour array and stylet

\section{Conclusions and Future Work}

In this work, we have designed and implemented a simulator that provides visual and haptic feedback during $\mathrm{CI}$ insertion into the ST. The simulation uses an anatomically accurate model of the ST, derived from measured data [2-5], and uses the Contour array for implantation. ST and BM models are parametric and may be easily reproduced for patient-specific data.

Outcomes of this work include manipulation of the virtual anatomy and electrode array using the haptic device interface, as well as force reflection provided through the device itself and quantified at the GUI. Specifically, stylet withdrawal and electrode array advancement into the ST with haptic feedback have been implemented.

The current simulator provides collision detection and response for only the tip of the array; and not the entire carrier. Future system design will include array curling during stylet withdrawal, with restoration forces exerted onto the ST walls. System validation by subjective analysis and experimentation (force measurement) is also needed. The final result will afford otologists with virtual training in $\mathrm{CI}$ insertion.

\section{References}

[1] C.A. Todd, F. Naghdy, S.J. O'Leary, "Geometric Modelling of a Haptic Rendered Computer Simulator for Cochlear Implantation", CIRAS 2003, Computer Conference Proceedings, Dec 2003.

[2] A. Kawano, H.L. Seldon, and G.M. Clark et al., "Computer-Aided Three-Dimensional Reconstruction in Human Cochlear Maps: Measurement of the Lengths of Organ of Corti, Outer Wall, Inner Wall, and Rosenthal's Canal", Ann. Otol. Rhinol. Laryngol., The Annals Publishing Co., USA, 1996, pp. (105):701-709.

[3] S-H. Hatsushika, R.K. Shepherd, and Y.C. Tong et al., "Dimensions of the Scala Tympani in the Human and Cat with Reference to Cochlear Implants", Ann. Otol. Rhinol. Laryngol., The Annals Publishing Co., USA, 1990, pp. (99):871-876.

[4] L.T. Cohen, J. Xu, and S.A. Xu et al, "Improved and Simplified Methods for Specifying Positions of the Electrode Bands of a Cochlear Implant Array", Am. J. Otol., The American Journal of Otology Inc., USA, 1996, pp. (17):859-865.

[5] D.R. Ketten, M.W. Skinner, and G. Wang et al., "In Vivo Measures of Cochlear Length and Insertion Depth of Nucleus Cochlear Implant Electrode Arrays", Ann. Otol. Rhinol. Laryngol., The Annals Publishing Co., USA, 1998, pp. (107, 11):1-16. 